\title{
Online Decentralized Perception-Aware Path Planning for Multi-Robot Systems
}

\author{
Nicola De Carli, Paolo Salaris, Paolo Robuffo Giordano
}

\begin{abstract}
This paper proposes a decentralized and online optimal perception-aware strategy for multi-robot systems. The aim is to maximize the information collected along the planned trajectory about the relative configurations of the robots and, hence, to minimize the localization uncertainty. This is done by leveraging the so-called Constructability Gramian (CG), which can quantify the information about the future state of a nonlinear system. We show that, thanks to a proper change of coordinates, the CG can be computed in a decentralized way with only minor approximations. This allows for formulating an online and decentralized trajectory generation problem for optimal localization. To show the effectiveness of the approach, we consider as case study the localization of a quadrotor group with noisy distance measurements and sensing constraints. The results show the interest of the proposed approach.
\end{abstract}

\section{INTRODUCTION}

Cooperative localization (CL) in a common frame from relative sensing is a classical topic in the multi-robot community. Many contributions have been proposed over the past years considering, for instance, under which conditions the localization problem can be solved (with different settings for the robot actuation, sensing and communication models), possible estimation schemes for recovering the relative positions/poses from the available measurements (considering, e.g., decentralization, explicit account of sensing/actuation noise, imperfect and/or intermittent communication, constraints such as sensing limitations, and so forth). For instance, in [1] an observability analysis for unicycle robots with different measurement models is proposed, while in [2] the same authors studied the estimation performances for the different measurement models using an Extended Kalman Filter (EKF). Numerous CL algorithms have been proposed using different estimation schemes [3]-[5], among which various instances of EKF [6]-[9].

Cooperative localization is, at its hearth, a nonlinear state estimation problem in which the states of interest (the relative poses) may or may not be observable depending on the particular configuration of the group (e.g, its current sensing/communication topology and the robot spatial arrangement) and/or the group motion (as in most nonlinear estimation problems, the trajectories followed by the system usually have a strong impact on the observability of its states). One possible way to address the localization problem that has gained attention over the last years is to leverage the notion of framework rigidity [10], [11] (or, more

N. De Carli and P. Robuffo Giordano are with CNRS, Univ Rennes, Inria, IRISA, Rennes, France. \{nicola.de-carli,prg\} @irisa.fr

P. Salaris is with the Research Center "E. Piaggio" and the Dept. of Information Engineering, University of Pisa, Pisa, Italy. paolo.salaris@ unipi.it simply, rigidity) for analysis and synthesis of localization algorithms. In the context of multi-robot localization, the notion of (infinitesimal) rigidity refers to the existence of a locally unique solution to the localization problem (modulo the usual unobservable global roto-translation of the whole group). Furthermore, whenever a formation is rigid, simple and decentralized localization algorithms can be designed, which are essentially based on a decentralized gradient descent. Some examples of the use of rigidity for cooperative localization can be found in [12]-[15] for the cases of distance and $3 \mathrm{D}$ bearing measurements.

Imposing rigidity to the robot formation can, however, be quite constraining since a sufficient number of edges per robot must be maintained at all times during motion. If presence of an edge is dictated by sensing/communication constraints (e.g., limited range or field-of-view), the rigidity requirement can clearly limit the group mobility especially in cluttered environments. It is worth noting that formation rigidity is only a sufficient condition for solving the localization problem which can also be solved without the rigidity condition but at the cost of introducing additional requirements on the group motion. When not rigid, the group must typically satisfy some persistent excitation condition [16], [17] for ensuring a converging localization (whereas no special robot motion is required in the rigid case). Clearly, the possibility of relaxing the rigidity requirement can be important during a mission for allowing better maneuverability and flexibility in the group shape (especially when coping with sensing/communication constraints) even if this imposes some constraints on the robot motion.

The main contribution of this paper is to propose a decentralized and online active sensing control strategy that optimizes the motion of each robot in the group for improving the localization accuracy. While several works have focused on multi-robot active sensing for target tracking [18]-[21] and for estimation of environmental quantities [22], [23], just few works consider the problem of active sensing to improve localization performance in a multi-robot system. In [24], it was considered the special case of holonomic vehicles, for which an analytical solution minimizing the trace of the covariance matrix given by the Riccati equation was provided. In [15], active sensing has been exploited but combined with bearing rigidity in order to retrieve an estimation of the scale of a formation of quadrotors. In order to relax the rigidity assumption, one needs to consider a measure of the collected information about the relative poses of the multirobot system during motion. To this end, in this work we extend to the multi-robot case the recently introduced notion 
of Constructability Gramian (CG) [25], and we detail an online decentralized trajectory optimization strategy able to generate trajectories that maximize the information about the final relative robot configurations. The proposed machinery is applied to a group of quadrotor UAVs with distance measurements and sensing constraints as case study, and the results show the effectiveness of the approach.

The rest of the paper is organized as follows: Section II introduces the main modeling assumptions and Sect. III recalls some relevant notions and properties of the CG. Section IV then presents one of the main results of this work, that is, a detailed analysis of the CG in a multi-robot setting and the proof of its decentralized structure once the 'right' coordinates are chosen for the group state. Subsequently Sect. V formulates the localization optimal control problem and discusses several possible norms for the CG to be used by the optimization. Section VI then presents statistical results on a number of simulations and Sect. VII concludes the paper.

\section{MULTI-ROBOT SYSTEM MODEL}

\section{A. Robot Model}

We consider a group of $N_{R}$ robots with a particular focus on quadrotor UAVs because of their popularity and widespread use in the community. However, the proposed methodology would also apply to other kinds of mobile robots with minor modifications.

As in related works on formation control/localization for multiple UAVs (e.g., [14], [26]-[28]), the UAV state is simplified into a 3D position and a yaw angle $\boldsymbol{q}_{i}=\left(\boldsymbol{p}_{i}, \psi_{i}\right) \in$ $\mathbb{R}^{3} \times \mathcal{S}^{1}$ which, as well-known, are the quadrotor flat outputs. Any smooth enough trajectory $\boldsymbol{q}_{i}(t)$ can then be tracked by any of the existing flight controllers for quadrotor UAVs (e.g., [29]). The UAVs are modeled as first-order kinematic systems with body-frame velocity commands

$$
\left[\begin{array}{c}
\dot{\boldsymbol{p}}_{i} \\
\dot{\psi}_{i}
\end{array}\right]=\left[\begin{array}{cc}
\boldsymbol{R}_{i} & \mathbf{0} \\
\mathbf{0} & 1
\end{array}\right]\left[\begin{array}{c}
\boldsymbol{v}_{i} \\
w_{i}
\end{array}\right]
$$

where $\boldsymbol{v}_{i}$ and $w_{i}$ are respectively the commanded body-frame linear velocity and yaw rate, and $\boldsymbol{R}_{i}=\boldsymbol{R}_{z}\left(\psi_{i}\right) \in S O(3)$ is the rotation matrix around the $z$-axis of an angle $\psi_{i}$. We also let $\boldsymbol{u}_{i}=\left(\boldsymbol{v}_{i}, w_{i}\right)$ collect the $i$-th robot commands.

No global common frame is assumed directly available to the robots since they can only obtain relative measurements w.r.t. each other and their velocity commands are in the robot body frames. A common frame must, however, be implicitly or explicitly selected for expressing the estimated robot poses. This could be an arbitrary 'fixed' frame (e.g., the initial pose of one particular robot in the group), or a 'moving' frame attached to the formation. In this latter case, two natural choices seem possible: the robot poses are expressed in the moving body frame of one particular robot (e.g., a leader or anchor), or in a moving frame attached to the group barycenter. The barycentral frame would seem a better choice since it does not require the selection of a 'special robot' in the group. However, being the barycentral frame linearly dependent on the frames of the robots in the formation, the associated change of coordinates would not be valid as the change of basis matrix would be rank deficient.

Therefore, in this work we assume presence of one leader/anchor robot, w.1.o.g. taken as robot 1 , whose body frame $\mathcal{A}_{1}$ is taken as the common frame for expressing the estimated poses of the other robots in the group. In view of the following developments, it is then convenient to express the robot states and model in terms of quantities relative to $\boldsymbol{A}_{1}$. Let ${ }^{1} \boldsymbol{p}_{i}={ }^{1} \boldsymbol{R}_{i}\left(\boldsymbol{p}_{i}-\boldsymbol{p}_{1}\right)$ and ${ }^{1} \psi_{i}=\psi_{i}-\psi_{1}$ be the position and orientation of robot $i$ in $\mathcal{A}_{1}$, and $\mathbf{x}_{i}=\left({ }^{1} \boldsymbol{p}_{i},{ }^{1} \psi_{i}\right)$ the $i$-th robot configuration in $\mathcal{A}_{1}$. From (1) and standard kinematics one has

$$
\dot{\mathbf{x}}_{i}=\left[\begin{array}{c}
{ }^{1} \dot{\boldsymbol{p}}_{i} \\
{ }^{1} \dot{\psi}_{i}
\end{array}\right]=\left[\begin{array}{cc}
{ }^{1} \boldsymbol{R}_{i} & \mathbf{0} \\
\mathbf{0} & 1
\end{array}\right]\left[\begin{array}{c}
\boldsymbol{v}_{i} \\
w_{i}
\end{array}\right]-\left[\begin{array}{cc}
\boldsymbol{I} & {\left[\mathbf{e}_{3}\right]_{\times}{ }^{1} \boldsymbol{p}_{i}} \\
\mathbf{0} & 1
\end{array}\right]\left[\begin{array}{c}
\boldsymbol{v}_{1} \\
w_{1}
\end{array}\right]
$$

where ${ }^{1} \boldsymbol{R}_{i}=\boldsymbol{R}_{z}\left({ }^{1} \psi_{i}\right), \mathbf{e}_{3}=\left[\begin{array}{lll}0 & 0 & 1\end{array}\right]^{T},[\cdot]_{\times}$is the skewsymmetric operator and $\boldsymbol{v}_{1}$ and $w_{1}$ are the control inputs of the leader. Clearly, ${ }^{1} \boldsymbol{p}_{1}={ }^{1} \dot{\boldsymbol{p}}_{1}=\mathbf{0}$ and ${ }^{1} \psi_{1}={ }^{1} \dot{\psi}_{1}=0$.

\section{B. Sensing and Communication Model}

The UAVs are assumed equipped with a sensor able to provide information about the relative pose of the robots. In the case of this work we consider range measurements corrupted by an additive normally distributed noise $\nu_{i j} \sim \mathcal{N}\left(0, r_{i j}\right)$ with zero mean and variance $r_{i j}$

$$
d_{i j}=\left\|\boldsymbol{p}_{j}-\boldsymbol{p}_{i}\right\|_{2}+\nu_{i j}=h_{i j}\left(\boldsymbol{p}_{j}-\boldsymbol{p}_{i}\right)+\nu_{i j} .
$$

Other measurements of interest (e.g., 3D bearings) are clearly possible and will be considered in future extensions of this work. Note that, in case of distance measurements, (3) can also be expressed in terms of quantities relative to the leader frame, i.e., $d_{i j}=h_{i j}\left({ }^{1} \boldsymbol{p}_{j}-{ }^{1} \boldsymbol{p}_{i}\right)+\nu_{i j}$. A robot pair $(i, j)$ is assumed able to measure $d_{i j}$ when $\left\|\boldsymbol{p}_{j}-\boldsymbol{p}_{i}\right\|_{2} \leq D_{u}$, with $D_{u}$ being a maximum sensing range. Similarly, a robot pair $(i, j)$ is also assumed able to exchange information over a radio channel when within the sensing range $D_{u}$. Again, more complex sensing/communication constraints can also be considered, see [30], [31] for some examples.

The group interaction is modeled with a directed sensing graph $\mathcal{G}(\mathcal{V}, \mathcal{E})$, where $\mathcal{V}=\left\{1, \ldots, N_{R}\right\}$ is the vertex set and $\mathcal{E} \subseteq \mathcal{V} \times \mathcal{V}$ the edge set. Presence of an edge $e_{k}=(i, j)$ represents the possibility for robot $i$ to obtain a measurement w.r.t. robot $j$. As usual, we let $\mathcal{N}_{i}$ be the set of all robots $j$ for which the 'forward' edge $(i, j)$ exists, and we also let $\mathcal{O}_{i}$ be the set of all robots $j$ for which the 'backward' edge $(j, i)$ exists (this distinction is needed because the graph is assumed directed). The communication graph is instead taken as the undirected counterpart of the sensing graph $\mathcal{G}$, i.e., two robots communicate if either $(i, j) \in \mathcal{E}$ or $(j, i) \in \mathcal{E}$. We note that the sensing model considered in this work (measurement available when $\left\|\boldsymbol{p}_{j}-\boldsymbol{p}_{i}\right\|_{2} \leq D_{u}$ ) would imply an undirected sensing graph. However, we prefer to keep our formulation general enough for accounting, in future extensions, for more complex directed sensing models (e.g., limited fov of onboard cameras). Moreover, because of the maximum range constraint, the sensing/communication graphs will obviously be time-varying with edges appearing/disappearing during the robot motion. 


\section{Group Model}

Let $\kappa$ be the state dimension of one robot $(\kappa=4$ in our case). In view of the following developments we let $\mathbf{x}=\left[\begin{array}{lll}\mathbf{x}_{2}^{T} & \ldots & \mathbf{x}_{N_{R}}^{T}\end{array}\right]^{T} \in \mathbb{R}^{\kappa\left(N_{R}-1\right)}$ represent the collection of the $N_{R}-1$ robot poses $\mathbf{x}_{i}$ in $\mathcal{A}_{1}$ (leader excluded), $\boldsymbol{u}=$ $\left[\boldsymbol{u}_{1}^{T}, \ldots, \boldsymbol{u}_{N_{R}}^{T}\right]^{T} \in \mathbb{R}^{\kappa N_{R}}$ the collection of all robot inputs, and $\boldsymbol{y}=\left[\begin{array}{lll}d_{1} & \ldots & d_{|\mathcal{E}|}\end{array}\right]^{T} \in \mathbb{R}^{|\mathcal{E}|}$ the collection of all the $|\mathcal{E}|$ distance measurements among robot pairs. Regrouping (2-3) for all the $N_{R}-1$ robots, the group dynamics in the leader frame is compactly expressed as

$$
\begin{array}{lll}
\dot{\mathbf{x}}(t) & =\boldsymbol{f}(\mathbf{x}(t), \boldsymbol{u}(t)), & \mathbf{x}\left(t_{0}\right)=\mathbf{x}_{0} \\
\boldsymbol{y}(t)=\boldsymbol{h}(\mathbf{x}(t))+\boldsymbol{\nu}(t) &
\end{array}
$$

where $\boldsymbol{\nu} \sim \mathcal{N}(0, \boldsymbol{R})$ collects all the measurement noise $\nu_{i j}$ with cumulative covariance matrix $\boldsymbol{R}=\operatorname{diag}\left(r_{i j}\right)$. Similarly, we also introduce vector $\boldsymbol{q}=\left[\begin{array}{lll}\boldsymbol{q}_{1}^{T} & \ldots & \boldsymbol{q}_{N_{R}}^{T}\end{array}\right]^{T} \in\left(\mathbb{R}^{3} \times \mathcal{S}^{1}\right)^{N_{R}}$ as the collection of configurations $\boldsymbol{q}_{i}$ of all the robots the global frame (this time leader included). Regrouping (1), we denote the group dynamics in the global frame as

$$
\dot{\boldsymbol{q}}(t)=\boldsymbol{g}(\boldsymbol{q}(t), \boldsymbol{u}(t)), \quad \boldsymbol{q}\left(t_{0}\right)=\boldsymbol{q}_{0} .
$$

This distinction between vectors $\mathbf{x}$ and $\boldsymbol{q}$ (and respective dynamics) may appear redundant but it will be important in the next developments: indeed $\mathbf{x}$ represents the 'leaderframe' robot poses that will be actually estimated by the employed filter (a EKF in our case), whereas $\boldsymbol{q}$ represents the 'fixed-frame' robot poses that are forward propagated by the robots at each optimization step for maximizing the future information gain. These points will be explained more clearly in Sect. V.

\section{INFORMATION MEASURES}

Among the many possible measures for quantifying the amount of information about the state of a nonlinear system, in this work we consider the so-called Gramians [32], [33] which have already been successfully applied to optimal estimation problems for single mobile robots [25], [34], [35]. Gramians indeed represent a general observability measure not related to the particular filter used for state estimation (e.g., a EKF). In particular, in this work we focus on the so-called Constructability Gramian (CG) rather than on the Observability Gramian (OG), since the CG provides a measure of the information collected during motion about the final state $\mathbf{x}_{f}$, while the OG measures the information collected about the initial state $\mathbf{x}_{0}$ [25]. We here briefly recall the main properties of the CG and its link with the OG which will be important in the next developments.

Consider the following quantities

$$
\begin{aligned}
& \mathbf{A}(t)=\frac{\partial \boldsymbol{f}(\mathbf{x}(t))}{\partial \mathbf{x}(t)} \in \mathbb{R}^{\kappa\left(N_{R}-1\right) \times \kappa\left(N_{R}-1\right)} \\
& \mathbf{H}(t)=\frac{\partial \boldsymbol{h}(\mathbf{x}(t))}{\partial \mathbf{x}(t)} \in \mathbb{R}^{|\mathcal{E}| \times \kappa\left(N_{R}-1\right)}
\end{aligned}
$$

and let $\boldsymbol{\Phi}\left(t, t_{0}\right) \in \mathbb{R}^{\kappa\left(N_{R}-1\right) \times \kappa\left(N_{R}-1\right)}$ be the so-called state sensitivity matrix. As well-known, matrix $\boldsymbol{\Phi}\left(t, t_{0}\right)$ obeys

$$
\dot{\boldsymbol{\Phi}}\left(t, t_{0}\right)=\boldsymbol{A}(t) \boldsymbol{\Phi}\left(t, t_{0}\right) \quad \boldsymbol{\Phi}\left(t_{0}, t_{0}\right)=\boldsymbol{I},
$$

and the following semigroup properties hold $\boldsymbol{\Phi}\left(t_{0}, t_{f}\right)=$ $\boldsymbol{\Phi}\left(t_{0}, \tau\right) \boldsymbol{\Phi}\left(\tau, t_{f}\right)=\boldsymbol{\Phi}^{-1}\left(\tau, t_{0}\right) \boldsymbol{\Phi}\left(\tau, t_{f}\right)$.

Let $\boldsymbol{W}(t) \in \mathbb{R}^{|\mathcal{E}| \times|\mathcal{E}|}$ be a symmetric positive definite weight matrix (a design parameter). By considering the weighted norm of the output variation $\delta \boldsymbol{y}(t)=\boldsymbol{y}(t)-\boldsymbol{y}^{o}(t)$, with $\boldsymbol{y}(t)$ the actual measurement and $\boldsymbol{y}^{o}$ the nominal one over a time interval $\left[t_{0}, t_{f}\right]$ due to a variation of the final state $\delta \mathbf{x}_{f}=\mathbf{x}\left(t_{f}\right)-\mathbf{x}^{o}\left(t_{f}\right)$, one has

$$
\left.\left\|\delta \boldsymbol{y}\left(t_{0}, t_{f}\right)\right\|_{\boldsymbol{W}(t)}=\delta \mathbf{x}_{f}^{T} \mathcal{G}_{c}\left(t_{0}, t_{f}\right)\right) \delta \mathbf{x}_{f}
$$

where $\mathcal{G}_{c}\left(t_{0}, t_{f}\right) \in \mathbb{R}^{\kappa\left(N_{R}-1\right) \times \kappa\left(N_{R}-1\right)}$ is the weighted CG defined as

$$
\mathcal{G}_{c}\left(t_{0}, t_{f}\right)=\int_{t_{0}}^{t_{f}} \boldsymbol{\Phi}^{T}\left(t, t_{f}\right) \boldsymbol{H}^{T}(t) \boldsymbol{W}(t) \boldsymbol{H}(t) \boldsymbol{\Phi}\left(t, t_{f}\right) d t .
$$

By letting $\boldsymbol{W}(t)=\boldsymbol{R}^{-1}(t)$ (the inverse of the output covariance matrix), the following important relationship holds between the weighted $\mathrm{CG}$ and the inverse of the optimal estimation error covariance matrix $\boldsymbol{P}(t)$ (as given by the Riccati equation) [25]

$$
\boldsymbol{P}^{-1}\left(t_{f}\right)=\boldsymbol{\Phi}^{T}\left(t_{0}, t_{f}\right) \boldsymbol{P}_{0}^{-1} \boldsymbol{\Phi}\left(t_{0}, t_{f}\right)+\mathcal{G}_{c}\left(t_{0}, t_{f}\right)
$$

where $\boldsymbol{P}_{0}=\boldsymbol{P}\left(t_{0}\right)$. This equation has the following interpretation: the first term represents the contribution of the a-priori information $\boldsymbol{P}_{0}^{-1}$ shifted at the final time $t_{f}$ by the operator $\boldsymbol{\Phi}\left(t_{0}, t_{f}\right)$, whereas the second term represents the information actually collected during the time interval $\left[t_{0}, t_{f}\right]$. If one further interprets $\boldsymbol{P}_{0}^{-1}$ as being the information collected over the past interval $\left(-\infty, t_{0}\right]$, equation (10) can be compactly expressed as $\boldsymbol{P}^{-1}\left(t_{f}\right)=\mathcal{G}_{c}\left(-\infty, t_{f}\right)$. Maximisation of a norm of $\mathcal{G}_{c}\left(-\infty, t_{f}\right)$ w.r.t. the state trajectory $\mathbf{x}(t)$ over $\left[t_{0}, t_{f}\right]$ is then expected to result in the minimization of a norm of the covariance matrix $\boldsymbol{P}\left(t_{f}\right)$ at the final time $t_{f}$ in addition to, more in general, improve the performance of the employed observer.

We conclude with the following additional decomposition of the $\mathrm{CG}$ which shows its link with the $\mathrm{OG}$ and, as explained in [25], is convenient for formulating an online optimal trajectory generation problem as the one considered in this work

$$
\mathcal{G}_{c}\left(-\infty, t_{f}\right)=\boldsymbol{\Phi}^{T}\left(t, t_{f}\right)\left(\mathcal{G}_{c}(-\infty, t)+\mathcal{G}_{o}\left(t, t_{f}\right)\right) \boldsymbol{\Phi}\left(t, t_{f}\right)
$$

where

$$
\mathcal{G}_{o}\left(t, t_{f}\right)=\int_{t}^{t_{f}} \boldsymbol{\Phi}^{T}(\tau, t) \boldsymbol{H}^{T}(\tau) \boldsymbol{R}^{-1}(\tau) \boldsymbol{H}(\tau) \boldsymbol{\Phi}(\tau, t) d \tau
$$

is the weighted Observability Gramian (OG). 


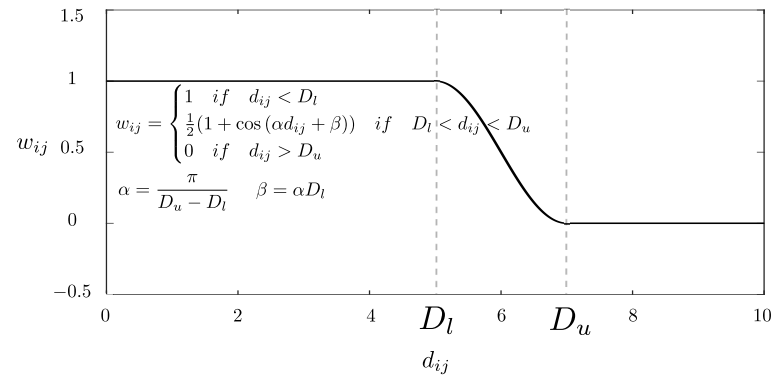

Fig. 1. Possible shape of the weights $w_{i j}$.

\section{GRAMIAN DECENTRALIZATION}

In this section, we provide one of the main contributions of this work, that is, the analysis of the structure of the CG and of its gradient w.r.t. the control inputs $\left(\boldsymbol{v}_{i}, w_{i}\right)$ of the $i$-th robot. We will show that, mainly thanks to a suitable change of coordinates for the robot states, it will be possible to obtain a decentralized structure for the CG and its gradient which will be instrumental for then solving the optimal estimation problem introduced in next Sect. V in a decentralized way.

\section{A. Structure of the $C G$}

In order to take into account the sensing/communication model of Sect. II-B, we introduce, as standard practice, a weight $w_{i j}\left(d_{i j}\right)$ for each edge $(i, j) \in \mathcal{E}$ which smoothly approaches zero as $d_{i j} \rightarrow D_{u}$. Fig. 1 shows a possible shape for this weight. By letting $\boldsymbol{W}_{e}=\operatorname{diag}\left(w_{i j}\right) \in \mathbb{R}^{|\mathcal{E}| \times|\mathcal{E}|}$, we define the weighted covariance matrix $\boldsymbol{R}_{W}$ to be used in (12) as $\boldsymbol{R}_{W}^{-1}=\boldsymbol{W}_{e}^{T} \boldsymbol{R}^{-1} \boldsymbol{W}_{e}$. With this choice if an edge is approaching disconnection because of the limited range $D_{u}$, the corresponding measurement covariance is forced to approach infinity indicating the loss of information over the edge.

As explained in the previous section, a norm of the CG $\mathcal{G}_{c}\left(-\infty, t_{f}\right)$, computed using the decomposition given in (11), is a suitable metric for formulating an online optimal trajectory generation problem. Possible norms for the CG will be discussed in Sect. V-A, while here we study in detail the structure of the CG in (11). We start by showing that the state sensitivity matrix $\boldsymbol{\Phi}$ is block diagonal: this can be easily shown by noting that, from the definition of $f$, one has

$$
\boldsymbol{A}=\frac{\partial \boldsymbol{f}}{\partial \mathbf{x}}=\left[\begin{array}{lll}
\boldsymbol{A}_{2} & & \\
& \ddots & \\
& & \boldsymbol{A}_{N_{R}}
\end{array}\right] .
$$

Since $\boldsymbol{\Phi}(t, t)=\boldsymbol{I}$ and $\boldsymbol{A}$ is block diagonal, from (7) it follows that

$$
\boldsymbol{\Phi}=\left[\begin{array}{lll}
\boldsymbol{\Phi}_{2} & & \\
& \ddots & \\
& & \boldsymbol{\Phi}_{N_{R}}
\end{array}\right]
$$

where each $\boldsymbol{\Phi}_{i} \in \mathbb{R}^{\kappa \times \kappa}$ is such that

$$
\dot{\boldsymbol{\Phi}}_{i}(\tau, t)=\boldsymbol{A}_{i}(\tau) \boldsymbol{\Phi}_{i}(\tau, t), \quad \boldsymbol{\Phi}_{i}(t, t)=\boldsymbol{I} .
$$

Let us now expand (11) as

$$
\begin{aligned}
\mathcal{G}_{c}\left(-\infty, t_{f}\right)= & \boldsymbol{\Phi}^{T}\left(t, t_{f}\right) \mathcal{G}_{c}(-\infty, t) \boldsymbol{\Phi}\left(t, t_{f}\right)+ \\
& +\boldsymbol{\Phi}^{T}\left(t, t_{f}\right) \mathcal{G}_{o}\left(t, t_{f}\right) \boldsymbol{\Phi}\left(t, t_{f}\right)=\boldsymbol{T}_{1}+\boldsymbol{T}_{2}
\end{aligned}
$$

and consider the first term $\boldsymbol{T}_{1}$. As explained in the previous section, one has $\mathcal{G}_{c}(-\infty, t)=\boldsymbol{P}^{-1}(t)$ where $\boldsymbol{P}(t)$ is the optimal estimation error covariance. In this work, an EKF is employed as state estimator so that a (converging) estimation of $\boldsymbol{P}(t)$ can be considered available at runtime. By approximating $\boldsymbol{P}(t)$ as block-diagonal, one can then conclude that $\boldsymbol{T}_{1}$ is also block-diagonal with $\kappa \times \kappa$ blocks

$$
\boldsymbol{T}_{1_{i i}}=\boldsymbol{\Phi}_{i}^{T}\left(t, t_{f}\right) \boldsymbol{P}_{i}^{-1}(t) \boldsymbol{\Phi}_{i}\left(t, t_{f}\right) \in \mathbb{R}^{\kappa \times \kappa} .
$$

Before considering the second term $\boldsymbol{T}_{2}$ we need to look at the structure of matrix $\boldsymbol{H}=\partial \boldsymbol{h} / \partial \mathbf{x}$ which is needed for evaluating (12). Using the measurement definition (3), it follows that the row of $\boldsymbol{H}$ associated to an edge $(i, j)$ takes the form

$$
\boldsymbol{H}_{i j}=\left[\begin{array}{lllllllll}
\mathbf{0} & \ldots & \underbrace{\boldsymbol{H}_{i j i}}_{i} & \ldots & \mathbf{0} & \ldots & \underbrace{\mathbf{H}_{i j j}}_{j} & \ldots & \mathbf{0}
\end{array}\right]
$$

with non-zero entries only on the $i$-th and $j$-th columns. Since the edge weights $w_{i j}$ are by construction zero for any nonneighboring pair of robots, after some (tedious) developments one can conclude that $\boldsymbol{T}_{2}$ can also be partitioned into $\kappa \times \kappa$ blocks with the non-zero blocks having expression

$$
\begin{aligned}
& \boldsymbol{T}_{2_{i j}}=\boldsymbol{\Phi}_{i}^{T}\left(t, t_{f}\right)\left(\int _ { t } ^ { t _ { f } } \boldsymbol { \Phi } _ { i } ^ { T } ( \tau , t ) \left(w_{i j}^{2}(t) \boldsymbol{H}_{i j i}^{T}(\tau) r_{i j}^{-1} \boldsymbol{H}_{i j j}(\tau)+\right.\right. \\
& \left.\left.+w_{j i}^{2}(\tau) \boldsymbol{H}_{j i i}^{T}(\tau) r_{i j}^{-1} \boldsymbol{H}_{j i j}(\tau)\right) \boldsymbol{\Phi}_{j}(\tau, t) d \tau\right) \boldsymbol{\Phi}_{j}\left(t, t_{f}\right), j \in \mathcal{N}_{i} \\
& \boldsymbol{T}_{2_{i i}}=\boldsymbol{\Phi}_{i}^{T}\left(t, t_{f}\right)\left(\int _ { t } ^ { t _ { f } } \boldsymbol { \Phi } _ { i } ^ { T } ( \tau , t ) \left(\sum_{k \in \mathcal{N}_{i}} w_{i k}^{2}(\tau) \boldsymbol{H}_{i k i}^{T}(\tau) r_{i k}^{-1} \boldsymbol{H}_{i k i}(\tau)\right.\right. \\
& \left.\left.\quad+\sum_{k \in \mathcal{O}_{i}} w_{k i}^{2}(\tau) \boldsymbol{H}_{k i i}^{T}(\tau) r_{k i}^{-1} \boldsymbol{H}_{k i i}(\tau)\right) \boldsymbol{\Phi}_{i}(\tau, t) d \tau\right) \boldsymbol{\Phi}_{i}\left(t, t_{f}\right), i=j .
\end{aligned}
$$

What can be concluded from this analysis is that matrix $\mathcal{G}_{c}\left(-\infty, t_{f}\right)$ has a natural 'decentralized' structure since every $\kappa \times \kappa\left(N_{R}-1\right)$ row block corresponding to robot $i$ consists of the diagonal block $\left(\boldsymbol{T}_{1_{i i}}+\boldsymbol{T}_{2_{i i}}\right)$ and of the off-diagonal blocks $\left(\boldsymbol{T}_{2_{i j}}\right)$ only for those $j \in \mathcal{N}_{i}$. In particular, the quantities needed by robot $i$ for evaluating 'its own' $i$-th block row are: the state sensitivity matrix $\boldsymbol{\Phi}_{i}$ and $\boldsymbol{\Phi}_{j}$ for $j \in \mathcal{N}_{i}$, the diagonal block $\boldsymbol{P}_{i}$ of the covariance matrix, the measurement noise variances $\left(r_{i j}, r_{k i}\right)$, the weights $\left(w_{i j}, w_{k i}\right)$ and the measurement matrices $\left(\boldsymbol{H}_{i j i}, \boldsymbol{H}_{i j j}, \boldsymbol{H}_{k i i}, \boldsymbol{H}_{k i k}\right)$ for $j \in$ $\mathcal{N}_{i}$ and $k \in \mathcal{O}_{i}$. Notice that the error covariance block $\boldsymbol{P}_{i}$, the measurement noise variances and the weights are locally available to robot $i$ or can be communicated by its 1-hop neighbors. However, the situation is not so favorable for what concerns the state transition matrices and measurement matrices (as well as the associated gradients) as discussed in the next section. 


\section{B. A Suitable Change of Coordinates}

Let us first consider the $i$-th $\kappa \times \kappa$ block of the state transition matrix $\boldsymbol{\Phi}_{i}$ : from (2) one has

$\boldsymbol{A}_{i}=\frac{\partial \boldsymbol{f}_{i}}{\partial \mathbf{x}_{i}}=\left[\begin{array}{cccc}0 & \omega_{1} & 0 & \left(-\sin \left({ }^{1} \psi_{i}\right) v_{i, x}-\cos \left({ }^{1} \psi_{i}\right) v_{i, y}\right) \\ -\omega_{1} & 0 & 0 & \left(\cos \left({ }^{1} \psi_{i}\right) v_{i, x}-\sin \left({ }^{1} \psi_{i}\right) v_{i, y}\right) \\ 0 & 0 & 0 & 0 \\ 0 & 0 & 0 & 0\end{array}\right]$

where $v_{i, x}$ and $v_{i, y}$ are the $x$ and $y$ components of the velocity input $\boldsymbol{v}_{i}$. Therefore, in order to implement (15) for evaluating $\boldsymbol{\Phi}_{i}(\tau, t)$ over the interval $\left[t, t_{f}\right]$, robot $i$ would require knowledge of its relative orientation ${ }^{1} \psi_{i}(t)$ in the leader frame and of the leader yaw rate $\omega_{\ell}(t)$ during the interval. These quantities may not be directly available if robot $i$ and the leader are not neighbors.

Furthermore, using the measurement definition (3), it follows that

$$
\begin{aligned}
& \boldsymbol{H}_{i j i}=\frac{\partial d_{i j}}{\partial \mathbf{x}_{i}}=\frac{1}{d_{i j}}\left[\left(\begin{array}{ll}
\left.{ }^{1} \boldsymbol{p}_{i}-{ }^{1} \boldsymbol{p}_{j}\right)^{T} & 0
\end{array}\right]\right. \\
& \boldsymbol{H}_{i j j}=\frac{\partial d_{i j}}{\partial \mathbf{x}_{j}}=\frac{1}{d_{i j}}\left[\left(\begin{array}{ll}
\left.{ }^{1} \boldsymbol{p}_{j}-{ }^{1} \boldsymbol{p}_{i}\right)^{T} & 0
\end{array}\right]\right. \text {. }
\end{aligned}
$$

As before, evaluation of $\boldsymbol{H}_{i j}$ by robot $i$ along the interval $\left[t, t_{f}\right]$ would require knowledge of the $i$-th and $j$-th robot position in the leader frame which may not be available if robots $i$ and/or $j$ are not neighbors of the leader, since the robots would need to know the future leader inputs in order to compute these quantities. Moreover, this coupling with the dynamics of the leader would also appear in the gradient computation, thus further complicating the optimization process since the leader inputs would be required for optimizing the CG blocks corresponding to each robot.

We then now show that it is indeed possible to avoid this inconvenient coupling thanks to a suitable change of coordinates. Consider the time-varying change of coordinates $\mathbf{x}(t)=\boldsymbol{T}(\boldsymbol{\psi}(t)) \mathbf{z}(t)$ where

$$
\boldsymbol{T}(\boldsymbol{\psi}(t))=\left[\begin{array}{lll}
\boldsymbol{T}_{2}\left({ }^{1} \psi_{2}(t)\right) & & \\
& \ddots & \\
& & \boldsymbol{T}_{N_{R}}\left({ }^{1} \psi_{N_{R}}(t)\right)
\end{array}\right]
$$

and

$$
\boldsymbol{T}_{i}\left({ }^{1} \psi_{i}(t)\right)=\left[\begin{array}{cc}
\mathbf{R}_{z}\left({ }^{1} \psi_{i}(t)\right) & \mathbf{0} \\
\mathbf{0} & 1
\end{array}\right] .
$$

One has $\delta \dot{\mathbf{x}}=\boldsymbol{T}(\boldsymbol{\psi}) \delta \dot{\mathbf{z}}+\frac{d}{d t} \boldsymbol{T}(\boldsymbol{\psi}) \delta \mathbf{z}$ and the linearized state equations can be re-written as

$$
\begin{aligned}
\delta \dot{\mathbf{z}}(t) & =\left[(\mathbf{T}(\boldsymbol{\psi}(t)))^{-1}\left(\mathbf{A}(t) \mathbf{T}(\boldsymbol{\psi}(t))-\frac{d}{d t} \mathbf{T}(\boldsymbol{\psi}(t))\right)\right] \delta \mathbf{z}(t)= \\
& =\tilde{\mathbf{A}}(t) \delta \mathbf{z}(t) \\
\delta \mathbf{y}(t) & =\mathbf{H}(t) \mathbf{T}(\boldsymbol{\psi}(t)) \delta \mathbf{z}=\tilde{\mathbf{H}}(t) \delta \mathbf{z}(t)
\end{aligned}
$$

where $\frac{d}{d t} \boldsymbol{T}(\boldsymbol{\psi}(t))$ is again a block diagonal matrix with each block expressed as

$$
\frac{d}{d t} \mathbf{T}_{i}(\boldsymbol{\psi})=\left[\begin{array}{cc}
\frac{d}{d t} \mathbf{R}_{z}\left({ }^{1} \psi_{i}\right) & \mathbf{0} \\
\mathbf{0} & 0
\end{array}\right]=\left[\begin{array}{cc}
\mathbf{R}_{z}\left({ }^{1} \psi_{i}\right)\left(\omega_{i}-\omega_{1}\right)\left[\mathbf{e}_{3}\right] \times & \mathbf{0} \\
\mathbf{0} & 0
\end{array}\right]
$$

Thus, in the new coordinates $\mathbf{z}$ the original matrix $\boldsymbol{A}$ is replaced by

$$
\tilde{\mathbf{A}}=\left[\begin{array}{lll}
\tilde{\boldsymbol{A}}_{2} & & \\
& \ddots & \\
& & \tilde{\boldsymbol{A}}_{N_{R}}
\end{array}\right]
$$

with each block having expression

$$
\begin{aligned}
\tilde{\mathbf{A}}_{i} & =\left(\mathbf{T}_{i}\left({ }^{1} \psi_{i}\right)\right)^{-1}\left(\mathbf{A}_{i} \mathbf{T}_{i}\left({ }^{1} \psi_{i}\right)-\frac{d}{d t} \mathbf{T}_{i}\left({ }^{1} \psi_{i}\right)\right)= \\
& =\left[\begin{array}{cccc}
0 & \omega_{i} & 0 & -v_{i, y} \\
-\omega_{i} & 0 & 0 & v_{i, x} \\
0 & 0 & 0 & 0 \\
0 & 0 & 0 & 0
\end{array}\right] .
\end{aligned}
$$

Notice that now $\tilde{\mathbf{A}}_{i}$ only depends on quantities relative to robot $i$. Similarly, in the new coordinates the original matrix $\boldsymbol{H}_{i j i}$ is replaced by

$$
\tilde{\mathbf{H}}_{i j i}=\mathbf{H}_{i j i} \mathbf{T}_{i}\left({ }^{1} \psi_{i}\right)=-\frac{1}{d_{i j}}\left[{ }^{i} \mathbf{p}_{j}{ }^{T} \quad 0\right]
$$

and analogously for $\boldsymbol{H}_{i j j}$, where ${ }^{i} \mathbf{p}_{j}$ denotes the position of robot $j$ expressed in the frame of robot $i$ with $j \in \mathcal{N}_{i}$. Again, the dependency on the leader is now avoided since $d_{i j}$ and ${ }^{i} \mathbf{p}_{j}$ can be evaluated from the sole knowledge of the future inputs of robot $i$ and robot $j$ that can be exchanged via 1 -hop communication.

Let then $\tilde{\boldsymbol{\Phi}}_{i}$ be the state transition matrix in the new coordinates obeying

$$
\dot{\tilde{\boldsymbol{\Phi}}}_{i}(\tau, t)=\tilde{\boldsymbol{A}}_{i}(\tau) \tilde{\boldsymbol{\Phi}}_{i}(\tau, t), \quad \tilde{\boldsymbol{\Phi}}_{i}(t, t)=\boldsymbol{I} .
$$

One can conclude that, in these coordinates, the relevant quantities $\tilde{\boldsymbol{\Phi}}_{i}, \tilde{\boldsymbol{H}}_{i j i}$ and $\tilde{\boldsymbol{H}}_{i j i}$ can be evaluated by robot $i$ over a time interval $\left[t, t_{f}\right]$ by only resorting to local or 1-hop information from its neihgbors, without the need of necessarily receiving information from the leader. In the new coordinates the $\mathrm{CG}$ is reformulated as

$$
\tilde{\mathcal{G}}_{c}\left(-\infty, t_{f}\right)=\tilde{\boldsymbol{\Phi}}^{T}\left(t, t_{f}\right)\left(\tilde{\mathcal{G}}_{c}(-\infty, t)+\tilde{\mathcal{G}}_{o}\left(t, t_{f}\right)\right) \tilde{\boldsymbol{\Phi}}\left(t, t_{f}\right)
$$

with

$$
\tilde{\mathcal{G}}_{o}\left(t, t_{f}\right)=\int_{t}^{t_{f}} \tilde{\boldsymbol{\Phi}}^{T}(\tau, t) \tilde{\boldsymbol{H}}^{T}(\tau) \boldsymbol{R}_{\boldsymbol{W}}^{-1}(\tau) \tilde{\boldsymbol{H}}(\tau) \tilde{\mathbf{\Phi}}(\tau, t) d \tau
$$

and

$$
\tilde{\mathcal{G}}_{c}(-\infty, t)=\tilde{\boldsymbol{P}}^{-1}(t)=\boldsymbol{T}(\hat{\boldsymbol{\psi}}(t)) \boldsymbol{P}^{-1}(t) \boldsymbol{T}(\hat{\boldsymbol{\psi}}(t))^{T} .
$$

Therefore, the diagonal blocks of $\tilde{\mathcal{G}}_{c}(-\infty, t)$ become $\tilde{\boldsymbol{P}}_{i}^{-1}=$ $\boldsymbol{T}\left({ }^{1} \hat{\psi}_{i}\right) \boldsymbol{P}_{i}^{-1} \boldsymbol{T}\left({ }^{1} \hat{\psi}_{i}\right)^{T}$, where ${ }^{1} \hat{\psi}_{i}(t)$ is the current estimated orientation of robot $i$ provided by the EKF (and, thus, locally available to robot $i$ at the current time $t$ ). The block decomposition detailed in (17-19) then becomes fully decentralized since each block can be evaluated by robot $i$ by only resorting to local and 1-hop information.

We finally note that, being $\boldsymbol{T}(\boldsymbol{\psi}(t))$ orthonormal, the proposed change of basis does not affect the spectral properties 
of the CG. In fact, using (8), one has in the new coordinates

$$
\begin{aligned}
& \left.\left\|\delta \boldsymbol{y}\left(t, t_{f}\right)\right\|_{\boldsymbol{R}_{W}^{-1}(\tau)}=\delta \boldsymbol{z}_{f}^{T} \tilde{\mathcal{G}}_{c}\left(t, t_{f}\right)\right) \delta \boldsymbol{z}_{f}= \\
& \left.=\left(\boldsymbol{T}\left(\boldsymbol{\psi}\left(t_{f}\right)\right)^{-1} \delta \mathbf{x}_{f}\right)^{T} \tilde{\mathcal{G}}_{c}\left(t, t_{f}\right)\right)\left(\boldsymbol{T}\left(\boldsymbol{\psi}\left(t_{f}\right)\right)^{-1} \delta \mathbf{x}_{f}\right)= \\
& \left.=\delta \mathbf{x}_{f}^{T}\left(\boldsymbol{T}\left(\boldsymbol{\psi}\left(t_{f}\right)\right) \tilde{\mathcal{G}}_{c}\left(t, t_{f}\right)\right) \boldsymbol{T}\left(\boldsymbol{\psi}\left(t_{f}\right)\right)^{-1}\right) \delta \mathbf{x}_{f}
\end{aligned}
$$

where the orthonormality of $\boldsymbol{T}(\boldsymbol{\psi}(t))$ has been used. Then by comparison with (8) it follows that

$$
\left.\left.\mathcal{G}_{c}\left(t_{0}, t_{f}\right)\right)=\boldsymbol{T}\left(\boldsymbol{\psi}\left(t_{f}\right)\right) \tilde{\mathcal{G}}_{c}\left(t_{0}, t_{f}\right)\right) \boldsymbol{T}\left(\boldsymbol{\psi}\left(t_{f}\right)\right)^{-1}
$$

showing that the $\mathrm{CG}$ in the new coordinates is similar to the $\mathrm{CG}$ in the original ones. Having the two matrices the same eigenvalues, optimizing some function of the eigenvalues of the CG in the new basis has the same effect as optimizing it in the original basis.

\section{MULTI-ROBOT OPTIMAL CONTROL PROBLEM}

Exploiting the analysis of the previous Section, the following trajectory optimization problem will determine the inputs $\boldsymbol{u}_{i}$ for each robot over a future time interval that maximize a norm of the $\mathrm{CG}$ and, hence, the information about the final group state.

Problem 1 (Multi-Robot Optimal Sensing Control)

For all $t \in\left[t_{0}, t_{f}\right]$, with $t_{f}$ fixed, find the optimal control strategy

$$
\begin{aligned}
& \boldsymbol{u}^{*}(t)=\underset{\boldsymbol{u}}{\operatorname{argmax}}\left\|\tilde{\mathcal{G}}_{c}\left(-\infty, t_{f}\right)\right\| \\
& \text { s.t. } \forall \tau \in\left[t, t_{f}\right] \\
& \boldsymbol{u}_{l} \leq \boldsymbol{u}(\tau) \leq \boldsymbol{u}_{u} \\
& \boldsymbol{y}(\tau) \geq \boldsymbol{y}_{l} \\
& \dot{\boldsymbol{q}}(\tau)=\boldsymbol{g}(\boldsymbol{q}(\tau), \boldsymbol{u}(\tau)) \\
& \boldsymbol{y}(\tau)=\boldsymbol{h}(\boldsymbol{q}(\tau), \boldsymbol{\nu}(\tau)) \\
& \dot{\tilde{\boldsymbol{\Phi}}}(\tau, t)=\tilde{\boldsymbol{A}}(\tau) \tilde{\boldsymbol{\Phi}}(\tau, t) \\
& \dot{\tilde{\mathcal{G}}}_{o}(t, \tau)=\tilde{\boldsymbol{\Phi}}^{T}(\tau, t) \tilde{\boldsymbol{H}}^{T}(\tau) \boldsymbol{R}^{-1}(\tau) \tilde{\boldsymbol{H}}(\tau) \tilde{\boldsymbol{\Phi}}(\tau, t) \\
& \tilde{\boldsymbol{\Phi}}\left(t_{f}, t\right)=\tilde{\boldsymbol{\Phi}}^{-1}\left(t, t_{f}\right) \\
& \tilde{\mathcal{G}}_{c}\left(-\infty, t_{f}\right)=\tilde{\boldsymbol{\Phi}}^{T}\left(t, t_{f}\right)\left(\tilde{\mathcal{G}}_{c}(-\infty, t)+\tilde{\mathcal{G}}_{o}\left(t, t_{f}\right)\right) \tilde{\boldsymbol{\Phi}}\left(t, t_{f}\right) \\
& \boldsymbol{q}(t)=(\mathbf{0}, \hat{\mathbf{x}}(t)), \tilde{\boldsymbol{\Phi}}(t, t)=\boldsymbol{I}, \tilde{\mathcal{G}}_{c}(-\infty, t)=\tilde{\boldsymbol{P}}^{-1}(t)
\end{aligned}
$$

where $\|\cdot\|$ is a suitable norm for the CG, $\boldsymbol{u}_{l}$ and $\boldsymbol{u}_{u}$ are minimum/maximum thresholds for the control inputs, and the constraint $\boldsymbol{y}(\tau) \geq \boldsymbol{y}_{l}$ aims at avoiding inter-agent collisions being $\boldsymbol{y}$ the vector of inter-agent distances and $\boldsymbol{y}_{l}$ a minimum allowed inter-agent distance ${ }^{1}$.

We note that, as expected, the relevant quantities needed for computing the CG over the future interval $\left[t, t_{f}\right]$ are expressed in the 'correct' coordinates which allows for a decentralized implementation by the robots in the group. Note also that the resolution of Problem 1 involves the forward integration over the future interval $\left[t, t_{f}\right]$ of several quantities (e.g., the state sensitivity matrix $\tilde{\boldsymbol{\Phi}}$ ), among which the group dynamics

\footnotetext{
${ }^{1}$ We note that this constraint only guarantees that robots connected by an edge will not collide. In the case studied in this work, any two robots closer than $D_{u}$ will be connected by an edge, and therefore the collision-avoidance constraint is always enforced. However, this could not be the case for other sensing modalities such as cameras with limited fov.
}

in the fixed frame (see (5) and discussion therein), which is initialized with the current available estimation from the EKF for all robots but the leader (line $\boldsymbol{q}(t)=(\mathbf{0}, \hat{\mathbf{x}}(t))$ ). Indeed, this initialization and the use of the dynamics (5) implicitly creates a 'fixed-frame', namely the frame of the leader at time $t$, where all the future robot poses are expressed (leader included). This allows any pair of neighboring robots to easily evaluate their relative position ${ }^{i} \boldsymbol{p}_{j}(\tau)$ over $\left[t, t_{f}\right]$ and, in turn, the evaluation of the various terms of the CG (see, e.g., (28)).

Remark 1. We remark that the employed EKF providing the estimated $\hat{\mathbf{x}}(t)$ and $\hat{\boldsymbol{P}}(t)$ is built on the 'leader-frame', dynamics (2). Therefore, implementation of the EKF requires that each robot has access to the current leader velocity input $\boldsymbol{u}_{1}(t)$ (see (2)). One possible way to address this issue is to assume presence of a broadcasting algorithm (e.g., a consensus-like propagation as the one used in [36]) for diffusing the current leader inputs to all the robots in the group at each estimation step. We also note that assuming presence of this propagation is conceptually different from requiring that every robot has access to the leader inputs (and relative poses) over the whole future interval $\left[t, t_{f}\right]$. In this latter case, the amount of information to be propagated to the group would be significant and could potentially grow very large as the future prediction horizon increases. This step is conveniently avoided thanks to the coordinate change presented in Sect. IV-B, and we instead only require propagation of the current leader velocity (a fixed, and small, amount of information per robot).

Remark 2. In order to reduce the computation and communication burden (w.r.t., e.g., a typical piecewise constant prameterization), in this work the inputs $\boldsymbol{u}_{i}$ are parameterized by $B$-splines using a fixed number of control points, which then effectively become the optimization variables of Problem 1. In this way, exploiting the convex hull of the control points, some constraints can be simplified [37] and the robots only need to exchange the control points of their future inputs and their current estimated state at each optimization iteration.

\section{A. Choice of the CG-based Metric}

Various possible norms for the Gramian have been considered in previous works, for instance the determinant of the inverse of the Gramian (also known as D-Optimality criterion); the trace of the inverse of the Gramian (AOptimality criterion); the condition number of the Gramian; and, finally, the minimum eigenvalue of the Gramian (EOptimality criterion). In the context of this work not all these possible metrics can result in a decentralized implementation of Problem 1, even though the CG itself has a decentralized structure. For example, any metric involving the inversion of the Gramian would not be decentralized. In this respect, a suitable metric for a decentralized implementation could be the trace of the $\mathrm{CG}$

$$
\left\|\tilde{\mathcal{G}}_{c}\left(-\infty, t_{f}\right)\right\|=\operatorname{trace}\left(\tilde{\mathcal{G}}_{c}\left(-\infty, t_{f}\right)\right) .
$$

Indeed, for optimizing the trace, each robot would simply need to optimize its own $i$-th and $j$-th blocks, $j \in \mathcal{N}_{i}$, of 
the CG without any dependency associated to other nonneighboring robots. However, a drawback of using the $\mathrm{CG}$ trace as a cost function is that its maximization does not have strong guarantees on the minimization of some norm of the estimation uncertainty [25].

Another possibility, which has shown to be more effective, is to consider the maximisation of the minimum eigenvalue of the CG that, although being a global quantity, could be estimated in a decentralized way by, e.g., adapting the algorithms presented in [13], [38]. However, the derivative of an eigenvalue is not well defined when the eigenvalue multiplicity is greater than one, and this (to the best of our knowledge) is still an open problem that can potentially degrade the performance of the eigenvalue estimation and its use in gradient-based optimization problems. A workaround to this issue has been proposed in [15] and consists in considering a smooth approximation of the minimum function by employing the generalized mean (without normalization) of the vector of eigenvalues [39]

$\left\|\tilde{\mathcal{G}}_{c}\left(-\infty, t_{f}\right)\right\|=\tilde{\lambda}_{m i n}\left(\tilde{\mathcal{G}}_{c}\left(-\infty, t_{f}\right)\right)=\sqrt[p]{\sum_{i=1}^{\kappa \cdot\left(\boldsymbol{N}_{R}-1\right)} \lambda_{i}^{p}\left(\tilde{\mathcal{G}}_{c}\left(-\infty, t_{f}\right)\right.}$

with $p \ll 0$. This solution is, however, not scalable since, as already pointed out in [30], it would require for each agent to estimate all the eigenvalues of the CG (and associated eigenvectors), whose number grows linearly with the size of the group.

In this work we then propose a scalable metric based on (36) as cost function, namely, the sum of the $\kappa \times \kappa$ diagonal blocks of $\tilde{\mathcal{G}}_{c}\left(-\infty, t_{f}\right)$

$$
\left\|\tilde{\mathcal{G}}_{c}\left(-\infty, t_{f}\right)\right\|=\sum_{i=1}^{N_{R}-1} \sqrt[p]{\sum_{j=1}^{\kappa} \lambda_{j}^{p}\left(\tilde{\mathcal{G}}_{c}^{i i}\left(-\infty, t_{f}\right)\right)}
$$

with $p \ll 0$, hereafter denoted as Sum of Approximated Minimum Eigenvalues (SAME). The use of (37) results in a decentralized optimization since each robot $i$ is only responsible for computing the inputs optimizing the approximated minimum eigenvalue of its $\kappa \times \kappa$ diagonal block of $\tilde{\mathcal{G}}_{c}$, whose evaluation is decentralized. The idea behind this metric is to minimize an approximation of the maximum uncertainty associated to the state of each robot whereas (36) aims at minimizing the uncertainty associated to the whole state of the system.

\section{SIMULATION RESULTS}

For illustration purposes, we first consider a group of $N_{R}=3$ robots that needs to localize itself using relative distance measurements, and we implement the decentralized EKF algorithm from [9] for estimating the robot states in a decentralized way. The measured distances are affected by gaussian noise with covariance $r_{i j}=0.3 \cdot T_{s} m^{2}$ where $T_{s}$ is the sampling period of the EKF. The initial state estimation $\hat{\mathbf{x}}\left(t_{0}\right)$ is affected by a gaussian noise with covariance $\sigma_{x y z 0}^{2}=0.4 m^{2}$ on the position and $\sigma_{\psi 0}^{2}=0.3 \mathrm{rad}^{2}$ on the yaw orientation. The thresholds defining the weights $w_{i j}$ (see Fig. 1) are $D_{l}=5 \mathrm{~m}$ and $D_{u}=7 \mathrm{~m}$. The inputs are

\begin{tabular}{|l|c|c|c|}
\hline Metric & $\lambda_{\max }\left(\boldsymbol{P}_{t_{f}}\right)$ & $\operatorname{trace}\left(\boldsymbol{P}_{t_{f}}\right)$ & $R M S E$ \\
\hline Random & 0.091548 & 0.12742 & 0.1286 \\
$\tilde{\lambda}_{\min }$ & 0.011747 & 0.017749 & 0.0362 \\
SAME & 0.019641 & 0.023787 & 0.0466 \\
\hline
\end{tabular}

TABLE I

AVERAGE OF THE MAXIMUM AND AVERAGE UNCERTAINTY AND RMSE AT $t_{f}$ FOR 3 ROBOTS

parameterized using B-Splines [40] with $N=6$ control points and their limits are set to $\boldsymbol{u}_{l}=-1$ and $\boldsymbol{u}_{u}=1$. Thanks to the B-spline parameterization, the robots can simulate the neighbors future trajectories by exchanging only a limited amount of information (the B-Spline control points), and they can also reduce the input constraints dimension considering the convex hull of the control points (in future we will consider also simplifications of the anti-collision constraints [37]).

Starting from 4 different fixed configurations, such that the initial sensing graph is connected, we generated 10 random paths of equal duration $t_{f}$ taken as initial guesses for Problem 1 which is then solved using either the SAME (37) or $\tilde{\lambda}_{\min }$ (36) metrics. Each trajectory (initial guess or optimized one) is executed 10 times with a different initial condition for the EKF drawn from a Gaussian centered at the true $\mathbf{x}\left(t_{0}\right)$ with covariance $\boldsymbol{P}_{0}$. When executing the initial nonoptimized trajectories, the sensing graph is considered fully connected (all relative measurements always available to the EKF) disregarding the sensing constraints, which are instead fully considered in the optimized cases (thus, resulting in a time-varying sensing graph). The final configuration $\mathbf{x}_{f}$ is not fixed while the final time is fixed at $t_{f}=15 \mathrm{~s}$.

The average of the maximum eigenvalue (corresponding to the maximum uncertainty) and of the trace (corresponding to the average uncertainty) of $\boldsymbol{P}\left(t_{f}\right)$, as well as the Root Mean Square Error (RMSE) at $t_{f}$ obtained optimizing the different metrics are compared and results are shown in Table I. One can note how, in average, the optimized cases perform better than the initial guesses .

One advantage of the proposed approach over a strategy based on instantaneous observability (like rigidity-based localization) is that the robot group has much more freedom in terms of mobility, which can be particularly useful when navigating in cluttered environments. In this respect Table II shows the results of a single illustrative run during which, for some optimized trajectories, the group does not remain rigid at all times because of the sensing constraints (the rigidity eigenvalue, a common measure of rigidity [13], is reported in Fig. 2). Despite the loss of rigidity, the group is still able to successfully localize and achieve good performance in terms of estimation accuracy (Table II). A video illustrating some representative trajectories for the multi-robot group is attached to the paper for the reader's convenience.

The same simulations have also been performed by considering $N_{R}=8$ robots and by using $r_{i j}=0.1 \cdot T_{s} m^{2}$ and $N=8$. The results are shown in Table III and show the good performance also in this case. The attached video allows to better appreciate the robot trajectories and behavior of the 


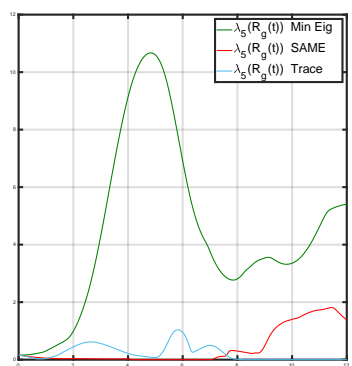

(a)

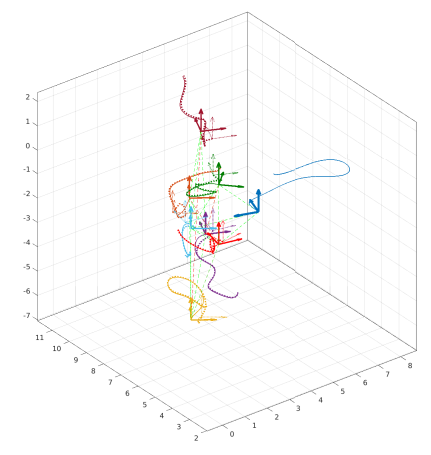

(b)

Fig. 2. An optimal trajectory which does not maintain the formation rigid. In 2(a), the rigidity eigenvalue is plotted. In 2(b) the robot trajectories are shown, with the frames in the initial positions (in blue is the leader) and in dotted lines the estimated trajectories.

\begin{tabular}{|l|c|c|c|}
\hline Metric & $\lambda_{\max }\left(\boldsymbol{P}_{t_{f}}\right)$ & $\operatorname{trace}\left(\boldsymbol{P}_{t_{f}}\right)$ & $R M S E$ \\
\hline Random & 0.0198620 & 0.0270547 & $2.1273 \mathrm{e}-04$ \\
$\tilde{\lambda}_{\text {min }}$ & 0.0002825 & 0.0014602 & $7.0901 \mathrm{e}-06$ \\
SAME & 0.0024085 & 0.0073659 & $1.3127 \mathrm{e}-04$ \\
Trace & 0.0512124 & 0.0550008 & $1.7742 \mathrm{e}-04$ \\
\hline
\end{tabular}

TABLE II

MAXIMUM AND AVERAGE UNCERTAINTY AND RMSE AT $t_{f}$ WITH SAME NOT PRESERVING RIGIDIY

estimation errors for this case study.

\section{CONCLUSIONS AND FUTURE WORKS}

In this paper, we have considered the localization problem for a multi-robot system using relative measurements between the robots. A decentralized formulation of the problem of active sensing control based on the optimization of a norm of the Constructability Gramian has been proposed. We have considered, as case study, a group of quadrotors measuring relative distances with maximum range constraints. The effectiveness of the proposed method has been shown by reporting the statistical results of several simulation runs.

Future works will aim at evaluating the computational times on the actual quadrotors and integrating the actuation noise (which for certain robots, e.g. quadrotors, is far from negligible) in the proposed scheme. Possible solutions could be based on the Riccati equation or on rechability concepts.

\begin{tabular}{|l|c|c|c|}
\hline Metric & $\lambda_{\max }\left(\boldsymbol{P}_{t_{f}}\right)$ & $\operatorname{trace}\left(\boldsymbol{P}_{t_{f}}\right)$ & $R M S E$ \\
\hline Random & 0.028253 & 0.041103 & 0.0621 \\
$\tilde{\lambda}_{\text {min }}$ & 0.00082939 & 0.0027855 & 0.0052 \\
SAME & 0.0087442 & 0.015267 & 0.0249 \\
Trace & 0.023022 & 0.029117 & 0.0513 \\
\hline
\end{tabular}

TABLE III

AVERAGE OF THE MAXIMUM AND AVERAGE UNCERTAINTY AND RMSE AT $t_{f}$ FOR 8 ROBOTS

Also, we want to consider the case in which the quadrotors are able to measure relative bearings obtained using an onboard camera and associated sensing constraints (e.g., limited fov). Furthermore, it could be interesting to evaluate the performance when the optimisation is constrained by higher priority tasks such as, e.g., reaching a final desired configuration or following a navigation plan.

\section{APPENDIX}

We here discuss how the structure of the CG in (19) (starting from the $\mathrm{OG}$ ) and the structure of the gradient of the CG in the new coordinates (29-30) w.r.t. the $i$-th robot inputs $\left(\boldsymbol{v}_{i}, w_{i}\right)$ have been obtained. We note that, as explained in [25], the term $\tilde{\mathcal{G}}_{c}(-\infty, t)$ in (29) is 'fixed' at the current time $t$, since it encodes the information collected over the past. Therefore, its gradient w.r.t. the future robot inputs is zero and one only need to focus on the terms $\tilde{\boldsymbol{\Phi}}\left(t, t_{f}\right)$ and $\tilde{\mathcal{G}}_{o}\left(t, t_{f}\right)$.

To study the structure of $\tilde{\mathcal{G}}_{o}\left(t, t_{f}\right)$ we start introducing an intermediary variable $\tilde{\boldsymbol{\Omega}}\left(t, t_{0}\right)=\boldsymbol{W}_{e}(t) \tilde{\boldsymbol{H}}(t) \tilde{\boldsymbol{\Phi}}\left(t, t_{0}\right)$. Then, $\tilde{\mathcal{G}}_{o}\left(t, t_{f}\right)$ can be rewritten as

$$
\tilde{\mathcal{G}}_{o}\left(t_{0}, t_{f}\right)=\int_{t_{0}}^{t_{f}} \tilde{\boldsymbol{\Omega}}^{T}\left(t, t_{0}\right) \boldsymbol{R}^{-1}(t) \tilde{\boldsymbol{\Omega}}\left(t, t_{0}\right) d t
$$

Considering a system of $N_{R}$ robots and $p$-dimensional outputs (in our case $p=1$ ) we have that $\Omega$ can be divided in $\frac{N_{R} !}{2 !\left(N_{R}-2\right) !}$ row-blocks (the possible combinations of two robots) and $\left(N_{R}-1\right)$ column-blocks, with blocks of dimension $p \times \kappa$. Each row-block corresponds to an edge $e_{k}=(i, j)$ and the column block $(i-1)$ corresponds to a robot $i$ (the jacobian w.r.t. the leader does not appear in $\boldsymbol{H}$ and thus in $\tilde{\mathbf{\Omega}}$ )

$$
\begin{aligned}
\tilde{\boldsymbol{\Omega}}^{(k, i-1)} & =w_{i j} \tilde{\boldsymbol{H}}_{i j i} \tilde{\boldsymbol{\Phi}}_{i} \\
\tilde{\boldsymbol{\Omega}}^{(k, j-1)} & =w_{i j} \tilde{\boldsymbol{H}}_{i j j} \tilde{\boldsymbol{\Phi}}_{j} \\
\tilde{\boldsymbol{\Omega}}^{(k, l)} & =\mathbf{0} \text { for } \quad l \neq(i-1), l \neq(j-1) .
\end{aligned}
$$

Since the covariance matrix $\boldsymbol{R}$ is block diagonal (diagonal in our case as $\boldsymbol{R}=\operatorname{diag}\left(r_{i j}\right)$ ), with each block corresponding to an edge,

$$
\begin{aligned}
\tilde{\mathcal{G}}_{o}^{i j} & =\int_{t_{0}}^{t_{f}} \tilde{\boldsymbol{\Phi}}_{i}^{T}\left(w_{i j}^{2} \tilde{\boldsymbol{H}}_{i j i}^{T} r_{i j}^{-1} \tilde{\boldsymbol{H}}_{i j j}+\right. \\
& \left.+w_{j i}^{2} \tilde{\boldsymbol{H}}_{j i i}^{T} r_{i j}^{-1} \tilde{\boldsymbol{H}}_{j i j}\right) \tilde{\boldsymbol{\Phi}}_{j} d t \quad \text { if } \quad i \neq j \\
\tilde{\mathcal{G}}_{o}^{i j} & =\int_{t_{0}}^{t_{f}} \tilde{\boldsymbol{\Phi}}_{i}^{T}\left(\sum_{k \in \mathcal{N}_{i}} w_{i k}^{2} \tilde{\boldsymbol{H}}_{i k i}^{T} r_{i k}^{-1} \tilde{\boldsymbol{H}}_{i k i}+\right. \\
& \left.+\sum_{k \in \mathcal{O}_{i}} w_{k i}^{2} \tilde{\boldsymbol{H}}_{k i i}^{T} r_{k i}^{-1} \tilde{\boldsymbol{H}}_{k i i}\right) \tilde{\boldsymbol{\Phi}}_{i} d t \quad \text { if } i=j .
\end{aligned}
$$




\section{REFERENCES}

[1] A. Martinelli and R. Siegwart, "Observability analysis for mobile robot localization," in 2005 IEEE/RSJ International Conference on Intelligent Robots and Systems. IEEE, 2005, pp. 1471-1476.

[2] A. Martinelli, F. Pont, and R. Siegwart, "Multi-robot localization using relative observations," in Proceedings of the 2005 IEEE international conference on robotics and automation. IEEE, 2005, pp. 2797-2802.

[3] A. Howard, M. J. Matark, and G. S. Sukhatme, "Localization for mobile robot teams using maximum likelihood estimation," in IEEE/RSJ international conference on intelligent robots and systems, vol. 1. IEEE, 2002, pp. 434-439.

[4] E. D. Nerurkar, S. I. Roumeliotis, and A. Martinelli, "Distributed maximum a posteriori estimation for multi-robot cooperative localization," in 2009 IEEE International Conference on Robotics and Automation. IEEE, 2009, pp. 1402-1409.

[5] D. Fox, W. Burgard, H. Kruppa, and S. Thrun, "A probabilistic approach to collaborative multi-robot localization," Autonomous robots, vol. 8, no. 3, pp. 325-344, 2000.

[6] S. S. Kia, S. Rounds, and S. Martinez, "Cooperative localization for mobile agents: A recursive decentralized algorithm based on kalmanfilter decoupling," IEEE Control Systems Magazine, vol. 36, no. 2, pp. 86-101, 2016.

[7] H. Li and F. Nashashibi, "Cooperative multi-vehicle localization using split covariance intersection filter," IEEE Intelligent transportation systems magazine, vol. 5, no. 2, pp. 33-44, 2013.

[8] L. C. Carrillo-Arce, E. D. Nerurkar, J. L. Gordillo, and S. I. Roumeliotis, "Decentralized multi-robot cooperative localization using covariance intersection," in 2013 IEEE/RSJ International Conference on Intelligent Robots and Systems. IEEE, 2013, pp. 1412-1417.

[9] L. Luft, T. Schubert, S. I. Roumeliotis, and W. Burgard, "Recursive decentralized collaborative localization for sparsely communicating robots." in Robotics: Science and Systems. New York, 2016.

[10] B. D. O. Anderson, Y. Changbin, B. Fidan, and J. Hendrickx, "Rigid graph control architectures for autonomous formations," IEEE Control Systems Magazine, vol. 6, no. 28, pp. 46-63, 2008.

[11] S. Zhao and D. Zelazo, "Bearing rigidity theory and its applications for control and estimation of network systems: Life beyond distance rigidity," IEEE Control Systems Magazine, vol. 39, no. 2, pp. 66-83, 2019.

[12] D. Zelazo, A. Franchi, and P. Robuffo Giordano, "Rigidity theory in $S E(2)$ for unscaled relative position estimation using only bearing measurements," in European Control Conference, ECC'14, 2014.

[13] D. Zelazo, A. Franchi, H. H. Bülthoff, and P. Robuffo Giordano, "Decentralized rigidity maintenance control with range measurements for multi-robot systems," The International Journal of Robotics Research, vol. 34, no. 1, pp. 105-128, 2015.

[14] F. Schiano, A. Franchi, D. Zelazo, and P. Robuffo Giordano, "A rigiditybased decentralized bearing formation controller for groups of quadrotor uavs," in 2016 IEEE/RSJ Int. Conf. on Intelligent Robots and Systems (IROS), 2016, pp. 5099-5106.

[15] R. Spica and P. Robuffo Giordano, "Active decentralized scale estimation for bearing-based localization," in IEEE/RSJ Int. Conf. on Intelligent Robots and Systems, 2016, pp. 5084-5091.

[16] G. Besançon, Nonlinear observers and applications. Springer, 2007, vol. 363 .

[17] P. Bernard, N. Mimmo, and L. Marconi, "On the semi-global stability of an ek-like filter," IEEE Control Systems Letters, 2020.

[18] R. Olfati-Saber, "Distributed tracking for mobile sensor networks with information-driven mobility," in 2007 American Control Conference. IEEE, 2007, pp. 4606-4612.

[19] P. Yang, R. A. Freeman, and K. M. Lynch, "Distributed cooperative active sensing using consensus filters," in Proceedings 2007 IEEE International Conference on Robotics and Automation. IEEE, 2007, pp. 405-410.

[20] T. H. Chung, J. W. Burdick, and R. M. Murray, "A decentralized motion coordination strategy for dynamic target tracking," in Proceedings 2006 IEEE International Conference on Robotics and Automation, 2006. ICRA 2006. IEEE, 2006, pp. 2416-2422.

[21] F. Morbidi and G. L. Mariottini, "Active target tracking and cooperative localization for teams of aerial vehicles," IEEE transactions on control systems technology, vol. 21, no. 5, pp. 1694-1707, 2012.

[22] D. Jang, J. Yoo, C. Y. Son, D. Kim, and H. J. Kim, "Multi-robot active sensing and environmental model learning with distributed gaussian process," IEEE Robotics and Automation Letters, vol. 5, no. 4, pp. 5905-5912, 2020.
[23] L. DeVries, S. J. Majumdar, and D. A. Paley, "Observability-based optimization of coordinated sampling trajectories for recursive estimation of a strong, spatially varying flowfield," Journal of Intelligent \& Robotic Systems, vol. 70, no. 1, pp. 527-544, 2013.

[24] A. Cristofaro and A. Martinelli, "Optimal trajectories for multi robot localization," in 49th IEEE Conference on Decision and Control (CDC). IEEE, 2010, pp. 6358-6364.

[25] P. Salaris, M. Cognetti, R. Spica, and P. Robuffo Giordano, "Online optimal perception-aware trajectory generation," IEEE Trans. on Robotics, vol. 35, no. 6, pp. 1307-1322, 2019.

[26] V. Mistler, A. Benallegue, and N. M'sirdi, "Exact linearization and noninteracting control of a 4 rotors helicopter via dynamic feedback," in Proceedings 10th IEEE international workshop on robot and human interactive communication. Roman 2001 (Cat. no. 01th8591). IEEE, 2001, pp. 586-593.

[27] D. Mellinger and V. Kumar, "Minimum snap trajectory generation and control for quadrotors," in 2011 IEEE international conference on robotics and automation. IEEE, 2011, pp. 2520-2525.

[28] A. Franchi, C. Masone, V. Grabe, M. Ryll, H. H. Bülthoff, and P. Robuffo Giordano, "Modeling and control of uav bearing formations with bilateral high-level steering," The International Journal of Robotics Research, vol. 31, no. 12, pp. 1504-1525, 2012.

[29] T. Lee, M. Leok, and N. H. McClamroch, "Geometric tracking control of a quadrotor uav on se (3)," in 49th IEEE conference on decision and control (CDC). IEEE, 2010, pp. 5420-5425.

[30] F. Schiano and P. Robuffo Giordano, "Bearing rigidity maintenance for formations of quadrotor uavs," in 2017 IEEE Int. Conf. on Robotics and Automation (ICRA), 2017.

[31] M. Aggravi, C. Pacchierotti, and P. Robuffo Giordano, "Connectivitymaintenance teleoperation of a uav fleet with wearable haptic feedback," IEEE Trans. on Automation Science and Engineering, 2021.

[32] A. J. Krener and K. Ide, "Measures of unobservability," in Proceedings of the 48h IEEE Conference on Decision and Control (CDC) held jointly with 2009 28th Chinese Control Conference. IEEE, 2009, pp. 6401-6406.

[33] B. T. Hinson and K. A. Morgansen, "Observability optimization for the nonholonomic integrator," in 2013 American Control Conference. IEEE, 2013, pp. 4257-4262.

[34] M. Cognetti, P. Salaris, and P. Robuffo Giordano, "Optimal active sensing with process and measurement noise," in 2018 IEEE Int. Conf. on Robotics and Automation, 2018, pp. 2118-2125.

[35] M. Cognetti, M. Aggravi, C. Pacchierotti, P. Salaris, and P. Robuffo Giordano, "Perception-aware human-assisted navigation of mobile robots on persistent trajectories," IEEE Robotics and Automation Letters, vol. 5, no. 3, pp. 4711-4718, 2020.

[36] A. Franchi and P. Robuffo Giordano, "Online leader selection for improved collective tracking and formation maintenance," IEEE Trans. on Control of Network Systems, vol. 5, no. 1, pp. 3-13, 2018.

[37] T. Mercy, W. Van Loock, and G. Pipeleers, "Real-time motion planning in the presence of moving obstacles," in 2016 European Control Conference (ECC). IEEE, 2016, pp. 1586-1591.

[38] P. Robuffo Giordano, A. Franchi, C. Secchi, and H. H. Bülthoff, "A passivity-based decentralized strategy for generalized connectivity maintenance," The Int. Journal of Robotics Research, vol. 32, no. 3, pp. 299-323, 2013.

[39] P. S. Bullen, Handbook of means and their inequalities. Springer Science \& Business Media, 2013, vol. 560.

[40] L. Biagiotti and C. Melchiorri, Trajectory planning for automatic machines and robots. Springer Science \& Business Media, 2008. 